\title{
ON THE DIVERSITY IN MULTIANTENNA HF COMMUNICATIONS
}

\author{
C. Peco*, S. Zazo ${ }^{\dagger}$, I. Pérez-Álvarez ${ }^{\#}$ J. López-Pérez ${ }^{\#}$ \\ ${ }^{*}$ Rohde \& Schwarz España, Dpto. Ingeniería de Sistemas. Madrid. Spain. Tel: +34-913340990 Ext 141, email: \\ carlos.peco@rohde-schwarz.com \\ ${ }^{\dagger}$ Universidad Politécnica de Madrid (UPM). Dpto. SSR. ETSI de Telecomunicación. Madrid. Spain. Tel.: +34-915495700 Ext \\ 4042, email: santiago@gaps.ssr.upm.es \\ \# Universidad de Las Palmas de Gran Canaria(ULPGC). CeTIC. Dpto. Señales y Comunicaciones. Las Palmas (Canary \\ Islands). Spain. Tel: +34-928457362, email: jlopez@cetic.eu
}

Keywords: HF, diversity, polarization, multiantenna, space.

\begin{abstract}
The objective of this paper is to contribute to one of the most challenging issues in modern HF communications: the exploitation of the multiantenna diversity and its impact in the need of time interleaving. Comparing two of the main diversity sources in multiantenna communications (the polarization and spatially separated antennas) we expect to empirically determine which is the best option as a trade off between performance and practical implementation issues, especially focused in the case where strict space constraints apply, as in naval and air platforms. In such applications, it is almost impossible to have antennas with enough physical separation to exploit some diversity. If the dual polarization processing proves itself as a reliable source of diversity, with just some additional hardware any existing system can be easily upgraded, improving the overall BER figures and/or reducing the delays imposed by the coders/interleavers. $[1,2,3]$
\end{abstract}

\section{Introduction}

Relying in the real HF link that our groups have established connecting the Canary Islands and Madrid (separated about $1800 \mathrm{~km}$ ), a recent addition to Madrid station makes it capable to analyze up to four simultaneous HF configuration, by means of four R\&S EK895 receivers and two dualpolarization R\&S HE016 antennas. In order to evaluate elliptically polarized signals, two phasing and combining networks are used, easily allowing the implementation of several configurations, depending of the experiment. The two antennas are 60 meters away of each other for space diversity studies, having one local (and fixed) and the other deployable at any distance between 0 and 60 meters by means of a special plattform. Our groups have been working in the HF field for a long time, and nowadays the equipment installed in the radio sites is fully controllable by a VPN network. $[4,5,6]$

\section{Comparison of Linear Vs Elliptical fields}

Our first approach to polarization diversity was the comparison of decorrelation obtained by means of linear vertical and linear horizontal polarization and the decorrelation obtained by means of right hand and left hand elliptical polarization. The exploitation of elliptical diversity needs additional circuitry to carefully combine both vertical and horizontal signals coming from the antennas, meaning cost and losses in the system, so there must be a real diversity gain when compared to linear diversity to incorporate this circuitry to a real setup.

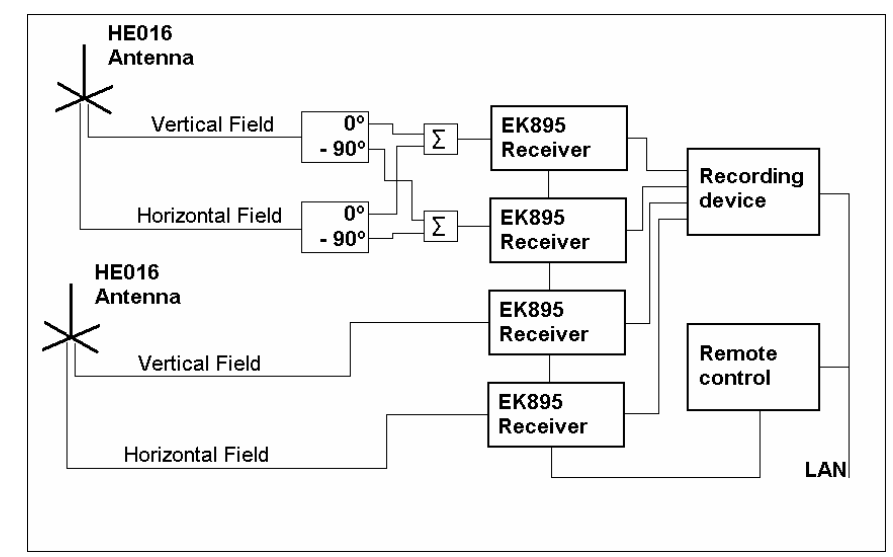

Figure 1. Configuration for simultaneous monitoring of four polarizations in two different antennas.

Next figure shows the evolution of the received signal (single $\mathrm{RF}$ tone evaluated in a $150 \mathrm{~Hz}$ bandwidth during 100 seconds) for the horizontal and vertical antenna (two first graphs) while the third graph shows how correlated the signals are. The highest correlation means that no advantages will be obtained from the diversity, while the lowest values shows the points where some gain will be obtained from the system. 

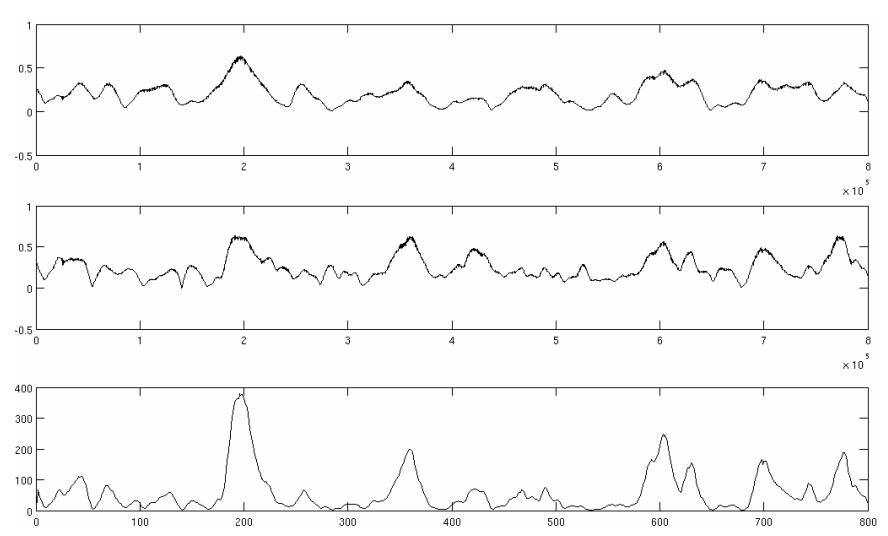

Figure 2. Horizontal and Vertical Polarization fields strength and its correlation over a 100 seconds period.

Correlation function is evaluated in 1000-samples slices, one slice every $1 / 8^{\text {th }}$ of second. For visualization purposes, it is not normalized.

Next figure shows the evolution of the same received signal (in the conditions described previously) for the right hand and left hand elliptical polarization (two first graphs) while the third graph shows how correlated the signals are. It can be observed (apart for the numerical computations) that there is no significant gain for the elliptical diversity when compared to the linear one.


Figure 3. Left and Right Elliptical Polarization fields strength and its correlation over a 100 seconds period.

\section{Comparison of Polarization Vs Space Diversity}

Having two separated away antennas is not an easy issue in naval or air platforms where there is a tight limitation in the space available, specially when dealing with large wavelengths. In our experimental setup we compared the decorrelation obtained with simple linear diversity (Vertical Vs Horizontal) with space diversity(Local Vertical Vs Remote Vertical). The frequencies employed for the tests were those to gave us at least two wavelengths of distance between antennas in a pure ionospheric path.
Next figure shows the evolution of the received signal (single $\mathrm{RF}$ tone evaluated in a $150 \mathrm{~Hz}$ bandwidth during 100 seconds) for the horizontal and vertical antenna (two first graphs) while the third graph shows how correlated the signals are.

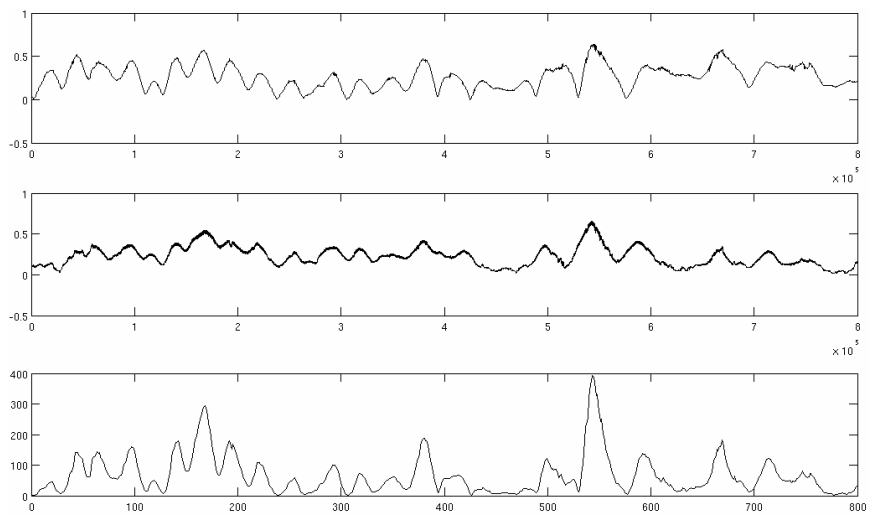

Figure 4. Horizontal and Vertical Polarization fields strength and its correlation over a 100 seconds period.

For the same transmitted signal, next figure shows the evolution of the same received signal (in the conditions described previously) local vertical antenna and remote vertical antenna (two first graphs) while the third graph shows how correlated the signals are. It can be observed (apart for the numerical computations) that the correlation is slightly higher in this case, meaning that the additional effort needed to have a second distant antenna for diversity purposes is not justified when compared with the simple, straightforward way of having an active antenna with dual polarization outputs.

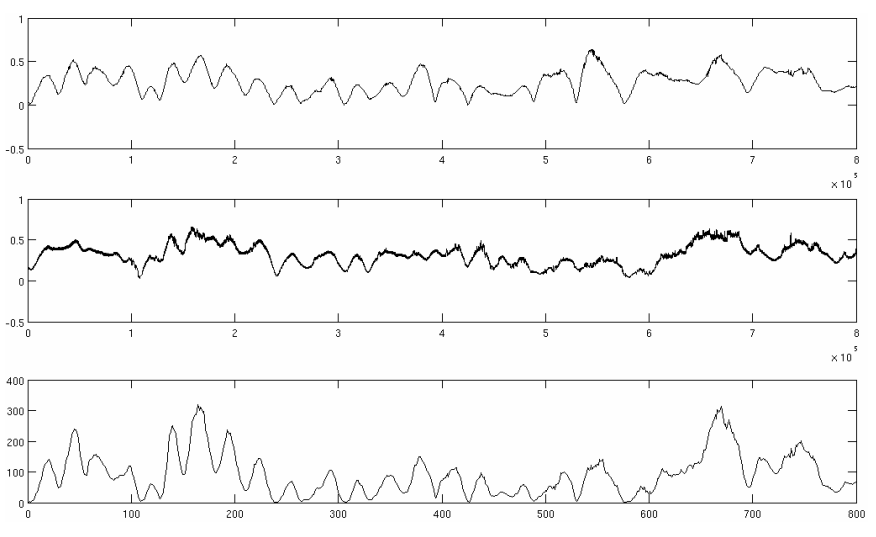

Figure 5. Local and Remote Antenna Vertical Polarization fields strength and its correlation over a 100 seconds period.

It can be guessed that the diversity gain that can be achieved by means of space diversity can be as significant (or perhaps even more significant) that the gain obtained by dual polarization techniques, but it needs an increase in terms of wavelengths separation that can not be accomplished in most of the cases. 


\section{Practical application of Diversity to Data Transmission}

Having acquired an overall idea on how to get the most from a HF diversity system with space constraints, the hypothesis can be confirmed by means of practical data transmission. Our goal was to confirm that, in the same way that the minima in the propagation channel took place in different times for each polarization, the errors in the decoded symbols will take place in different times for each polarization, too. In the same way, we were expecting a practical confirmation that, in terms of diversity, the highest error correcting capabilities can be obtained by linear polarization diversity in a single antenna system rather than in a complex space diversity system.

The setup to perform this test is quite simple: the remote transmitter (Canary Islands, $1800 \mathrm{~km}$ away) transmits a known pattern of characters in BPSK at low latency (31 bps) and the received signal by means of different receivers and antennas is recorded for off-line demodulation and analysis.

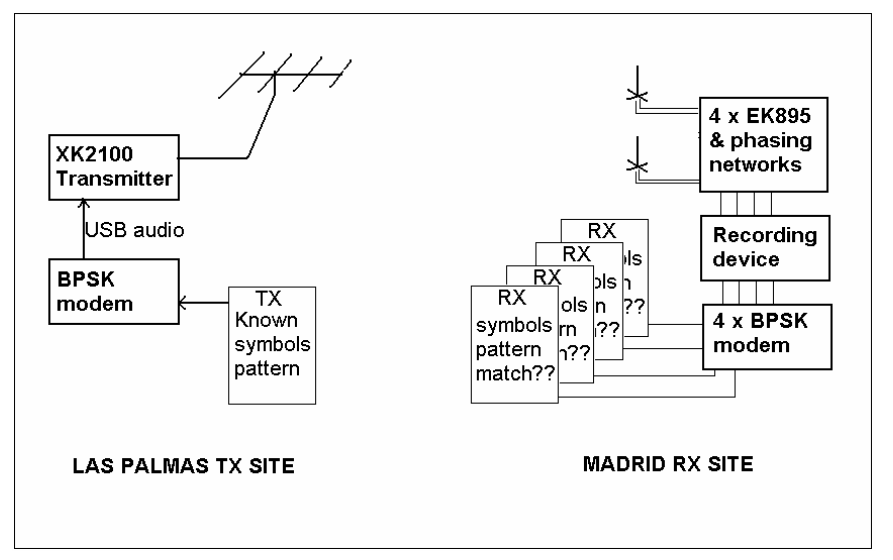

Figure 6. Simplified block diagram of the setup.

Next figure shows (in a 1024 symbols sequence) when the errors in symbol recovery are made, having represented in the first graph the errors of the modem for the vertical field as "ones", and the errors (also as "ones") of the modem for the horizontal field in the second one. It can be seen that the errors take place in different moments, so it is easy to perform automatic corrections where a bit or symbol error detection is available.

As stated before, the transmission is done using a file containing a known pattern of source-symbols, so the evaluation of wrongly decoded symbols is a straightforward process involving a symbol by symbol comparison.
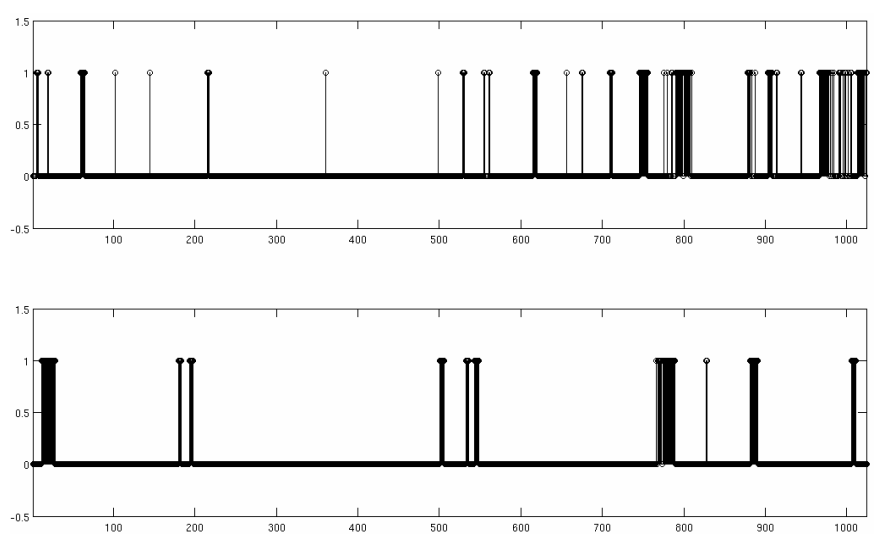

Figure 7. Errors located in the received symbols pattern for Horizontal and Vertical Polarizations.

Note that the number of errors associated to the horizontal antenna is slightly higher than those associated to the vertical one; this effect is due to a poorer SNR ratio in the horizontal antenna, which is receiving a large amount of man-made noise coming from the building where is installed. The vertical antenna (even being located in the same place) has a different radiation pattern and is much less sensitive to the noise coming underneath; this man-made noise (coming from computers and other devices) can ruin any experiment if it is not taken into account.

Next figure shows (in the first graph) the cases where an error was associated to the horizontal field or associated to the vertical field (an XOR operation), meaning that in these cases the errors could be fixed by means of diversity. The second row represents the cases where an error was made in both receiving channels, meaning that no error correction can be performed by the solely use of diversity resources. In these cases additional coding is needed, but it can be guessed that the size of this coder will be smaller if the polarization diversity is exploited.
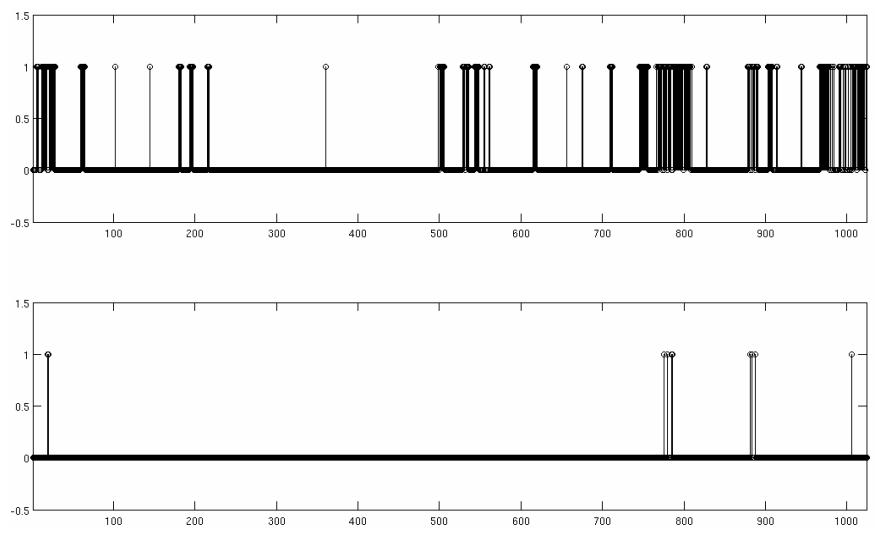

Figure 8. Errors made in one Polarization channel that might be fixed, and errors made in both polarizations.

In order to confirm that linear polarization diversity exhibits slightly better results than space diversity (for distances between one and three wavelengths), the same experiment 
was repeated receiving both vertical local and vertical remote fields. After offline processing, the results are shown in the following figure:
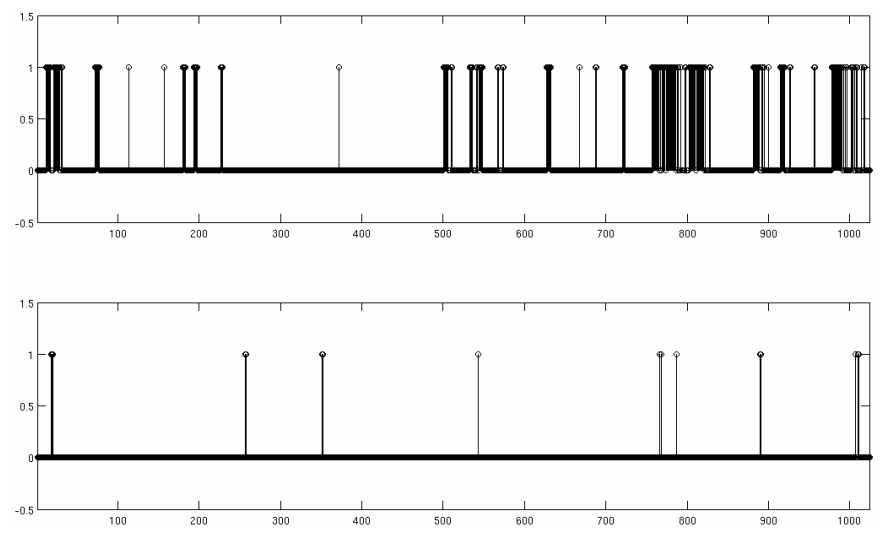

Figure 9. Errors made in Vertical Local or Vertical Remote antenna that might be fixed, and unrecoverable errors made in both polarizations.

\section{Conclusions}

This paper shows a set of preliminary tests done in order to determine the optimum configuration for a polarization and space diversity for HF links. The main goal is to reduce the delay imposed by the channel coders while maintaining the BER. Our results show that the benefits of linear polarization diversity are approximately the same than those obtained by elliptical polarization diversity and greater than those obtained by cumbersome space diversity where space and budget constraints.

\section{Acknowledgements}

We would like to thank Aena for their support and also the Spanish Education and Science Ministry under Grant TEC2007-67520-C02-01/02/TCM

\section{References}

[1] A. Bisiaux, L. Bertel, "Antenna Diversity as a means of Improving HF Transmissions" Proceedings of 2001 Antenna Applications Symposium, Septiembre 2000

[2] A. Bisiaux, D. Lemur, L. Bertel, “ Collocated-Antenna Arrays: Application to Digital Communications in HF”, IEEE Antennas and Propagation Society International Symposium, Julio 2003

[3] C. Perrine, D. Lemur, A. Bourdillon, Y. Erherl, N. Melida Garrido "1300km HF Radio Link with a $30 \mathrm{kbits} / \mathrm{s}$ Data Transfer Rate",Tenth IEE International Conference on Ionospheric Radio Systems and Techniques (IRST06), Julio 2006
[4]Santana, H.; Zazo, S.; Pérez, I.; Raos, I.; Mendieta, E.; López, J. Validation of a HF Spread Spectrum MultiCarrier Technology through real Link Measurements. European Trans. on Telecommunications (ETT), Vol17,Nov.-Dec. 2006

[5] Santana-Sosa, H.; Pérez-Álvarez, I.; A.; Mendieta-Otero, E.; López-Pérez, J.;Zazo, S.; Raos, I.; Performance over a real link of a HF software radio modem for interactive digital voice communications. HF 2006: Tenth International Conference on HF Radio Systems and Techniques 2006, London, UK, July 2006

[6]Zazo, S.; F. Bader; J.M Paez Borrallo. Spread Spectrum OFDM Modem for HF Voice-Band Link Over Fading Channels. European Transactions on Telecommunications, Vol.11, Nov.-Dec. 2000 